\title{
Transcriptional activity of Pannier is regulated negatively by heterodimerization of the GATA DNA-binding domain with a cofactor encoded by the u-shaped gene of $\mathrm{D}$ rosophila
}

\author{
Marc Haenlin, Yolande Cubadda, Francois Blondeau, Pascal Heitzler, Yves Lutz, Pat Simpson, ${ }^{1}$ and \\ Philippe Ramain ${ }^{1}$ \\ Institut de Génétique et de Biologie Moléculaire et Cellulaire (IGBMC), Centre N ational de la Recherche Scientifique/ \\ Institut N ational de la Santé et de la Recherche M édical e/Université Louis Pasteur (CN RS/IN SERM /ULP), 67404 IIIkirch \\ Cedex, Communauté U rbaine de Strasbourg, France
}

The genes pannier (pnr) and u-shaped (ush) are required for the regulation of achaete-scute during establishment of the bristle pattem in D rosophila. pnr encodes a protein belonging to the GATA family of transcription factors, whereas ush encodes a novel zinc finger protein. Genetic interactions between dominant pnr mutants bearing lesions situated in the amino-terminal zinc finger of the GATA domain and ush mutants have been described. We show here that both wild-type Pannier and the dominant mutant form activate transcription from the heterologous $\alpha$ globin promoter when transfected into chicken embryonic fibroblasts. Furthermore, Pnr and Ush are found to heterodimerize through the amino-terminal zinc finger of Pnr and when associated with Ush, the transcriptional activity of Pnr is lost. In contrast, the mutant pnr protein with lesions in this finger associates only poorly with Ush and activates transcription even when cotransfected with Ush. These interactions have been investigated in vivo by overexpression of the mutant and wild-type proteins. The results suggest an antagonistic effect of Ush on Pnr function and reveal a new mode of regulation of GATA factors during development.

[Key Words: Drosophila; pannier; u-shaped; heterodimerization; GATA; DN A-binding domain; bristle pattern]

Received July 1, 1997; revised version accepted August 27, 1997.

The GATA factors comprise a family of transcription factors that interact specifically with the (A/T)GATA(A) $G)$ consensus sequence through a highly conserved zinc finger DN A-binding domain (Wall et al. 1988; Evans and Felsenfeld 1989; Tsai et al. 1989; Orkin 1992). At present, six members of this family have been identified in birds that have homologs in mammals and amphibians (Laverierre et al. 1994). GATA-binding proteins have also been isolated from fungi, yeast, flies, and worms (Kudla et al. 1990; Cunningham and Cooper 1991; Spieth et al. 1991; A bel et al . 1993; Ramain et al. 1993; Winnick et al . 1993; Lin et al. 1995).

The founding member of this family, GATA-1, was identified originally as an erythroid cell DNA-binding

${ }^{1}$ Corresponding authors.

E-MAIL psimpson@titus.u-strasbgfir; phr@titus.u-strasbg.fr; FAX (33) 3 88653201 . factor that interacts with the promoters and enhancers of many erythroid-specific genes (Evans and Felsenfeld 1988). Targeted disruption of GATA-1 in mice revealed that this protein is needed for the maturation of terminally differentiated erythroblasts (Pevny et al. 1991). High levels of expression of gl obin genes require regulatory sequences, di spersed over a wide area, that appear to cooperate with each other to drive globin expression (Crossley and Orkin 1993; Higgs and Wood 1993; Andrews and Orkin 1994). This cooperation is thought to involve protein-protein interactions and indeed two proteins that associate with GATA-1 have been described: Sp1, which is expressed ubiquitously, and the Krüppellike factor EKLF, which is specific to erythroid cells (Miller and Bieker 1993; Merika and Orkin 1995). These proteins heterodimerize through their respective DNAbinding domains and the carboxy-terminal zinc finger of GATA-1 is sufficient to mediate physical association (Merika and Orkin 1995). When associated with GATA- 
1, Spl and EKLF have a synergistic effect on transcription.

Previously, we described the molecular cloning of pannier (pnr), a gene from Drosophila whose product bears a putative DNA-binding domain with two zinc fingers that are homologous to those of vertebrate GATA-1 (Ramain et al. 1993). pnr is required for the spatial regulation of the achaete and scute genes during bristle patterning in Drosophila. achaete and scute (sc) encode proteins bearing a basic helix-loop-helix motif and are required to provide cells with neural potential (Villares and Cabrera 1987; Ghysen and Dambly-Chaudière 1988; Campuzano and Modolell 1992). They are expressed in restricted groups of cells, the proneural clusters, at the sites where the macrochaete (large bristle) precursors form (Romani et al. 1989; Cubas et al. 1991; Skeath and Carroll 1991; Cubas and M odolell 1992). Mutants of pnr display changes in the number and positions of bristles that are correlated with changes in ac-sc expression (Ramain et al. 1993; Heitzler et al. 1996).

We show here that Pannier binds to the GATA core sequence and we have investigated some of its transcriptional properties making use of its ability to activate the heterologous $\alpha$-globin promoter when transfected into chicken embryonic fibroblasts. One class of dominant pnr alleles is associated with point mutations causing a single amino acid change in the amino-terminal zinc finger (Ramain et al. 1993). In spite of these lesions, the mutant proteins also activate transcription in the transient expression assay. In vivo, however, the mutants display an increase of ac-sc expression and additional bristles on the thorax, but decreased ac-sc expression and a loss of bristles at other sites (Ramain et al. 1993; Heitzler et al. 1996). This suggests that additional factors, differentially distributed within the epithelium, may regulate the activity of Pnr during development.

We have identified another gene, u-shaped (ush), mutants of which interact genetically with the dominant pnr mutants (Heitzler 1993; Cubadda et al., this issue). Mutants of ush display additional thoracic bristles. Lowering the dosage of ush enhances, whereas increasing the dosage suppresses, the phenotype of flies heterozygous for the alleles of pnr-bearing point mutations in the amino-terminal zinc finger. ush encodes a protein containing nine zinc fingers (five $\mathrm{C}_{2} \mathrm{HC}$ fingers and four $\mathrm{C}_{2} \mathrm{H}_{2}$ fingers) clustered in the amino and carboxyl termini of the protein (Cubadda et al., this issue). We show here that $U$ sh and Pnr dimerize and that this interaction is mediated by the amino-terminal zinc finger of Pnr. Presumably because they bear lesions in this motif, association of the mutant forms of Pnr with Ush is severely reduced. When coexpressed with $U$ sh in the transient expression assay, activity of the wild-type, but not the mutant, form of Pnr is strongly antagonized. The consequences of overexpression of U sh and the different forms of Pnr on an ac-lacZ reporter in transgenic flies are consistent with an antagonistic effect of Ush on the activity of Pnr in the regulation of ac-sc expression and bristle development. Thus, we have identified a new cofactor for a GATA homolog that mediates its effects through protein-protein interactions involving the amino-terminal zinc finger, unlike the previously identified Spl and EKLF that associate with the carboxy-terminal zinc finger.

\section{Results}

Pnr binds to the GATAAG consensus sequence and activates the $\alpha$-globin promoter in chicken embryonic fibroblasts

To determine a preferential binding sequence for Pnr, a random pool of degenerate oligonucleotides was screened for binding affinity to an immobilized fusion protein between glutathionine S-transferase (GST) and the Pnr DN A-binding domain (GST-Pnr-DBD) on a glutathione-agarose column (see $M$ aterials and Methods). The bound oligonucleotides were subcloned into the pBluescript SK + plasmid after four cycles of selection and PCR amplification. Of the 29 clones sequenced, 27 were found to contain a single GATA motif, and two exhibited a GATA repeat (Fig. 1A). Alignment of the different sequences allowed us to derive the consensus sequence 5'-GATAAG-3' (Fig. 1B). When the enriched pool was used in an electrophoretic mobility-shift assay (EMSA), two specific complexes were detected (Fig. 1C). The slower migrating one could be either oligonucleotides bearing a repeat of the GATA sequence with a single GST -Pnr-DBD molecule bound to each motif (Fig. $1 A$ ) or to a dimer of the GST -Pnr-DBD protein bound to a single GATA sequence. We have shown that Pnr, like GATA-1, is able to homodimerize through its GATADBD (Crossley et al 1995; R. Woehl and P. Ramain, unpubl.).

The consensus GATA sequence defined for Pnr binding is identical to that present in the $\alpha$-globin promoter recognized by the chicken GATA-1 protein (cGATA-1). This promoter is active throughout development in erythroid cells and is unlikely to be regulated by stage- and tissue-specific factors. Furthermore, it can be activated by CGATA-1 after transfection in a chicken embryonic fibroblast (CEF) cell line (Evans and Felsenfeld 1991). To investigate transcriptional activity of Pnr we used a transient expression assay in CEF cells with a Pnr expression vector and a reporter in which the chloramphenicol acetyltransferase (CAT) sequences are under the control of the wild-type $\alpha$-gl obin promoter. Figure 2B shows that expression of either CGATA-1 or Pnr stimulates activity of the $\alpha$-gl obin reporter 35-fold. The effect of Pnr is mediated through a repeat of the GATA motif present in the promoter, as mutation of both GATA sequences abolishes activity (data not shown). Furthermore, experiments using mutated or multimerized GATA sequences from the globin gene upstream of an AdhCAT reporter gene (a minimal promoter from the Drosophila alcohol dehydrogenase gene) show that, like cGATA-1, Pnr binds as a monomer to the proximal GATA motif to stimulate transcription (data not shown). This is consistent with the consensus-binding sequence that we defined (Fig. $1 C)$, since the distal GATA motif contains a $G$ in posi- 


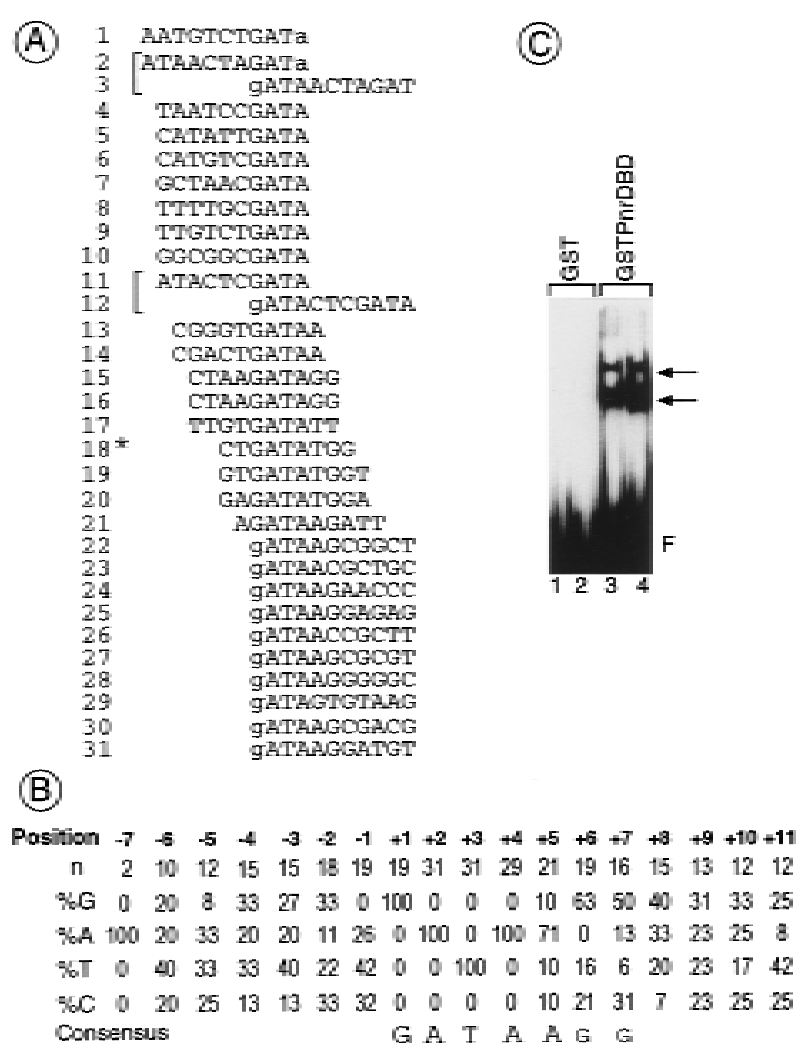

Figure 1. Definition of a consensus-binding site for Pnr. Degenerated ol igonucl eotides were loaded on a GST column where a fusion protein between the GST and the Pnr-DBD is bound (GST-Pnr-DBD). After elution, the selected oligonucleotides were PCR amplified and loaded again on the column (see Materials and Methods for details). After four cycles of selection and PCR amplification, the oligonucleotides were both subcloned for sequence analysis and used as a template in an electrophoretic mobility-shift assay (EMSA). (A) Alignment of 29 Pnr binding sequences recovered after four rounds of selection. The nucleotides included in the random region are shown in uppercase letters, whereas the bordering $\mathrm{G}$ and $\mathrm{A}$ nucleotides are in lowercase letters. The sequences are al igned with respect to the GATA motif. Brackets indicate ol igonucl eotides containing a repeat of the GATA sequence. The asterisk $\left(^{*}\right)$ denotes a template containing only nine nucleotides in the random region, which may correspond to either incomplete oligonucleotide synthesis or to PCR artifacts. (B) Consensus-binding site derived from the frequencies of the bases at the sites selected. (C) Autoradiography of an EMSA using as a template the pool of ol igonucleotides sel ected and as a protein extract either purified GST (Ianes 1,2: 20 and 200 ng, respectively) or GST-Pnr-DBD (lanes 3,4: 20 and $200 \mathrm{ng}$, respectively). (F) Unbound oligonucleotides. The arrows indicate two complexes of which the one migrating more sl owly may correspond either to a template containing two GATA sequences (A) with one molecule bound to each motif or to a dimer bound to a template containing a single GATA sequence.

tion -1 ; therefore, it does not correspond to the target sequence for binding of Pnr.

Previously we have described two mutant forms of the Pnr protein (Ramain et al. 1993). The dominant alleles
$\mathrm{pnr}^{\mathrm{D} 1}, \mathrm{pnr}^{\mathrm{D} 2}, \mathrm{pnr}^{\mathrm{D} 3}$, and $\mathrm{pnr}^{\mathrm{D} 4}$ (collectively called $\mathrm{pnr} \mathrm{r}^{\mathrm{D}}$ ) are associated with point mutations resul ting in proteins with a single amino acid change in the amino-terminal zinc finger. They are associated with an overexpression of ac-sc and ectopic dorsocentral bristles on the thorax. In contrast, the alleles $\mathrm{pnr}^{\mathrm{V} \times 1}$ and $\mathrm{pnr}^{\mathrm{V} \times 4}$ (collectively
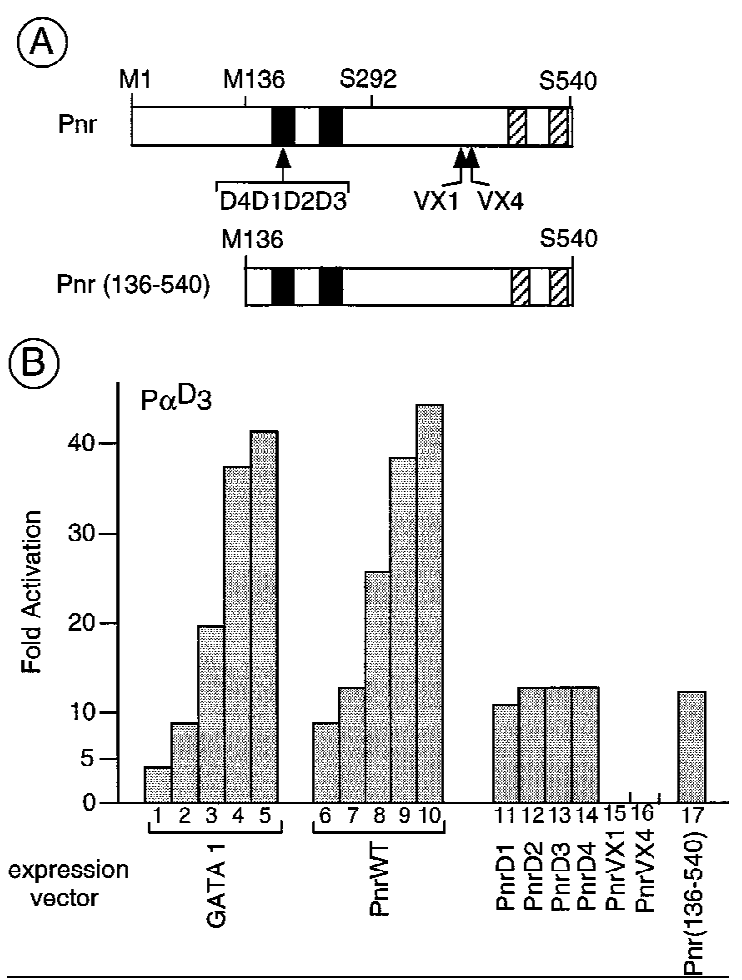

Figure 2. Transactivation of the $\alpha$-globin promoter sequences by different forms of Pnr in chicken embryonic fibroblasts (CEF). (A) Structural features of the different pnr proteins used in the present study indicating the two zinc fingers (solid boxes) and two sequences organized as putative amphipathic $\alpha$ helices (hatched boxes). The point mutations associated with the $\mathrm{pnr}^{\mathrm{D}}$ al lel es that result in a single amino acid exchange are located in the amino zinc finger, whereas the lesions associated with the $p^{\vee} r^{\vee \times 1 / 4}$ alleles and corresponding to a frameshift mutation in the open reading frame are localized in the amino-terminal of the two amphipathic helices. The amino acids are indicated with the single letter code and numbering refers to that given in Ramain et al. (1993). The methionine-136 (M 136) is used as an internal start codon in the truncated protein Pnr (136-540) encoded by the pXJ(Sph-N ot) expression vector (see M aterials and M ethods). In contrast, the chimeric proteins (see Fig. 4) contain either the amino acids methionine-1 to serine-292 (chimera Pnr-TEF-1) or the amino acids serine-292 to serine-540 (chimera Gal4-Pnr). (B) Transactivation of the $\alpha$-globin promoter by the different forms of Pnr. The CEF cells were cotransfected with the $\alpha$-globin reporter $(6 \mu \mathrm{g}$ of $\mathrm{P} \alpha \mathrm{D} 3)$ and the expression vector for CGATA-1 (lanes 1-5; 25, 50, 100, 250, and 500 ng), wild-type

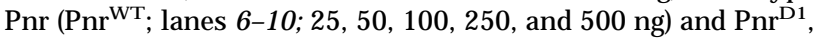
$\mathrm{Pnr}^{\mathrm{D} 2}, \mathrm{Pnr}^{\mathrm{D} 3}, \mathrm{Pnr}^{\mathrm{D} 4}, \mathrm{Pnr}^{\mathrm{VX} 1}, \mathrm{Pnr}^{\mathrm{VX} 4}$, and Pnr (136-540), (lanes 11-17: 50 ng each). The level of activation is expressed relative to the reporter alone and as the average (S.D. $\pm 20 \%$ ) of three independent experiments performed with two independent DNA preparations. 
called $\mathrm{pnr}^{\mathrm{V} \times 1 / 4}$ ) are characterized by a frameshift deletion in the coding sequences deleting the two amphipathic $\alpha$-helices present in the carboxyl terminus of the protein. They are associated with decreased ac-sc expression and a loss of dorsocentral bristles. The activity of these mutant proteins in the transient expression assay was tested.

The Pnr ${ }^{\vee \times 1 / 4}$ proteins that contain a wild-type DNAbinding domain do not activate the $\alpha$-globin promoter, suggesting the loss of an activation domain in the carboxyl part of the protein (Fig. 2B, lanes 15,16; see bel ow). In contrast, we found that the $\mathrm{Pnr}^{\mathrm{D}}$ forms of the protein, with a mutated DBD, activate the $\alpha$-globin promoter as efficiently as the wild type (Fig. 2B, cf. lane 7 and lanes 11-14). This is consistent with in vitro observations showing that they interact with the GATA motif of the $\alpha$-globin promoter in an EMSA (data not shown). In addition we also found that the amino part of Pnr can be removed without affecting activity (Fig. 2B, cf. Ianes 7 and 17; see below).

U sh negatively regulates activation by Pnr but not by $\mathrm{Pnr}^{\mathrm{D}}$

The ush gene encodes a large zinc finger protein that al so affects ac-sc expression and the number of bristles (Cubadda et al., this issue). The phenotype of $\mathrm{pnr}^{\mathrm{D}}$, but not pnr $r^{\vee 1 / 4}$, heterozygotes is sensitive to the amount of Ush present. The number of ectopic bristles in $\mathrm{pnr}^{\mathrm{D}} /+$ mutants increases in flies bearing only a single copy of ush $^{+}$, but decreases when three copies are present (Cubadda et al., this issue). Activation of the $\alpha$-globin promoter sequences in CEF cells by Pnr was used as an assay to study the effects of the U sh protein on the function of Pnr. When both Ush and wild-type Pnr are expressed simultaneously by cotransfection, activation of the promoter is abol ished. Stimulation by Pnr is lost progressi vely in a concentrati on-dependent manner (Fig. 3A, lanes 1-4, 8-11). Similarly, activation by CGATA-1 is also lost after cotransfection with the Ush expression vector (Fig. 3A, lanes 12-14). Because Pnr and cGATA-1 have no homol ogy outsi de their GATA-DBD, and as U sh al one has no effect on globin promoter activity (data not shown), these observations suggest that the function of Ush is mediated through the GATA-DBD.

To further investigate the interaction between Ush and the GATA-DBD-containing molecules, we used two chimeric proteins. In the first, the activation domain of transcriptional enhancer factor-1 (TEF-1), a Simian virus 40 enhancer-binding factor (Xiao et al. 1991; Hwang et al. 1993), is fused to the Pnr-GATA-DBD, and in the second, the carboxyl terminus of Pnr is fused to the Gal4DBD (constructs Pnr-TEF-1 and Gal4-Pnr, respectively; Fig. 4). When expressed in CEF, the Pnr-TEF-1 fusion protein stimulates the globin promoter sequences. Cotransfection with a U sh expression vector, however, reduces this activation in a concentration-dependent manner (Fig. 4B, lanes 1-4). The Gal4-Pnr chimeric protein stimulates activity of the 17m5-TATA-CAT reporter, in which the CAT gene is under the control of five Gal4-
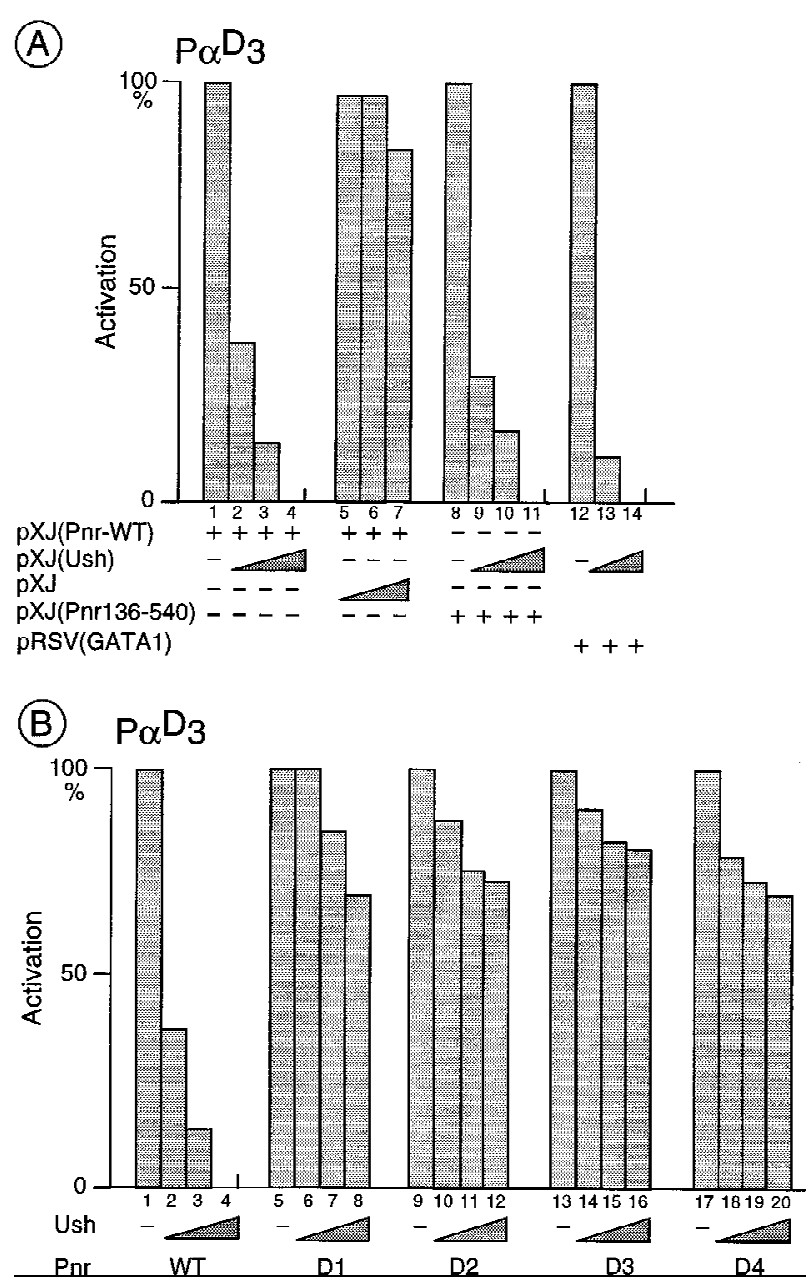

Figure 3. In vivo interactions between Ush and Pnr or CGATA- 1 in CEFs. (A) Activation of the $\alpha$-globin promoter sequences by CGATA-1 and wild-type Pnr is reduced strongly in the presence of Ush. CEF cells were cotransfected with the $\alpha$ gl obin reporter ( $6 \mu \mathrm{g}$ of $\mathrm{P} \alpha \mathrm{D} 3)$ and an expression vector either for the full-length Pnr (lanes 1-7, 40 ng), or the truncated Pnr (136540) (lanes 8-11, 40 ng), or CGATA-1 (lanes 12-14, 40 ng) and increasing amounts of an expression vector for U sh (lanes 2, 9, 13: 20 ng; lanes 3, 10, 14: 40 ng; lanes 4, 11: 80 ng). Cotransfection with the empty pXJ expression vector (lanes 5-7: 20, 40, and $80 \mathrm{ng}$, respectively) is used as a control. The CAT activities are expressed relative to the Pnr and CGATA-1 expression vectors al one, where the CAT activity is fixed arbitrarily at $100 \%$. In each case, they represent the average (S.D. $\pm 20 \%$ ) of three independent experiments performed with two independent DNA preparations. (B) Activation of the $\alpha$-globin promoter sequences by the mutant protein $\mathrm{Pnr}^{\mathrm{D}}$ is affected poorly by $\mathrm{U}$ sh. CEF cells are cotransfected with the $\alpha$-globin reporter (6 $\mu \mathrm{g}$ of $P \alpha D 3)$, an expression vector for either the wild-type Pnr or one of the mutated $\mathrm{Pnr}^{\mathrm{D}}$ proteins (40 ng in each case; lanes 1-4: wild-type Pnr; lanes 5-8: PnrD1; lanes 9-12: PnrD2; lanes 13-16: $\mathrm{Pnr}^{\mathrm{D} 3}$; lanes 17-20: $\mathrm{Pnr}^{\mathrm{D} 4}$ ) and increasing amounts of a Ush expression vector (lanes 2,6,10,14,18: 20 ng; Ianes 3,7,11,15,19: $40 \mathrm{ng}$; lanes 4,8,12,16,20: $80 \mathrm{ng}$ ). The activation represents the average (S.D. $\pm 20 \%$ ) of three independent experiments performed with two independent DNA preparations and is expressed as a percentage of the full activation (100\%) seen with Pnr alone. 

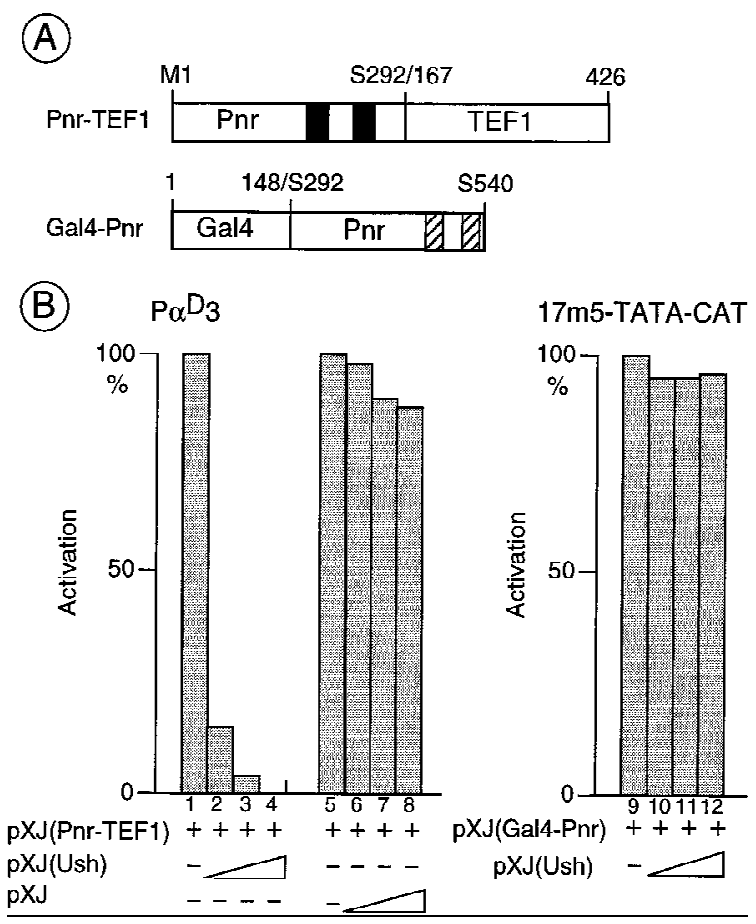

Figure 4. In vivo interactions between Ush and Pnr-DBD containing chimeric proteins. (A) Structural features of the chimeras used in the present study. The Pnr-TEF-1 chimera results from a fusion of the amino terminus of Pnr (M 1-S292) including the DNA-binding zinc fingers (solid boxes), and the amino acids 167-426 of the SV40-enhancer binding protein TEF-1 that bears an activation function. The Gal4-Pnr chimera results from a fusion of the Gal4-DNA-binding domain (amino acids 1-148) and the carboxyl terminus of Pnr (S292-S540) containing the two $\alpha$ helices (hatched boxes). (B) Specific inhibition by Ush of the transcriptional activation induced by the Pnr-DBD-containing chimera. CEFs were cotransfected with either the $\alpha$-gl obin reporter $(6 \mu \mathrm{g}$ of $P \alpha D 3)$, or the upstream activating sequence (UAS) reporter ( $1 \mu \mathrm{g}$ of $17 \mathrm{m5}$-TATA-CAT), an expression vector encoding either the Pnr-TEF-1 (lanes 1-8: 40ng), or the Gal 4-Pnr chimera (lanes 9-12: 40ng), and increasing amounts of a U sh expression vector (lanes 2,10: 20ng; Ianes 3,11: 40ng; Ianes 4,12: 80ng). Cotransfection of the empty pXJ expression vector (lanes 6-8: 20, 40, and 80 ng, respectively) is used as a control. The activation represents the average (S.D. $\pm 20 \%$ ) of three independent experiments performed with two independent DN A preparations and is expressed as a percentage of the full activation $(100 \%)$ seen with the chimera alone.

binding sites, indicating the presence of an activating function in the carboxyl terminus. However, stimulation is not affected by cotransfection of the U sh expression vector (Fig. 4B, lanes 9-12). Therefore, Ush affects specifically stimulation by the proteins containing a GATA-DBD.

Despite bearing amino acid substitutions in the amino terminal zinc finger, the $\mathrm{Pnr}^{\mathrm{D}}$ proteins stimulate the globin promoter as efficiently as wild-type Pnr (see Fig. 2B). Because it was shown earlier that the phenotype of the $\mathrm{pnr}^{\mathrm{D}}$ heterozygotes is sensitive to the amount of ush $^{+}$ product, we looked for a possible interaction between
Ush and these proteins. In contrast to the wild type, whose activity is reduced strongly by the presence of Ush, the $\mathrm{Pnr}{ }^{\mathrm{D}}$ proteins are poorly sensitive in this assay; stimulation by $\mathrm{Pnr}^{\mathrm{D}}$ is not notably reduced when cotransfected with Ush (see Fig. 3B, cf. Ianes 1-4 with lanes 5-8, 9-12, 13-16, and 17-20). Therefore, it is likely that the amino acids that have been mutated in $\mathrm{Pnr}^{\mathrm{D}}$ are re quired for a mol ecular interaction between Pnr and Ush.

Pnr heterodimerizes with U sh through its GATA-DBD

As U sh can antagonize transcriptional activation by Pnr, we then addressed the question as to whether there is a physical association between these two proteins. Protein extracts were made from Cos cells cotransfected with expression vectors for Pnr and a tagged U sh (see M aterials and M ethods). The two wild-type proteins coimmunoprecipitate and could be detected on Western blots with appropriate antibodies (Fig. 5A). In contrast, the $\mathrm{Pnr}^{\mathrm{D}}$ proteins with mutated GATA-DBDs are only weakly associated with Ush in similar experiments (Fig. 5 A, cf. lanes 3-6 with lane 2). This suggests that the association of the two proteins requires the amino-terminal zinc finger.

The interaction between Ush and Pnr was al so tested in yeast. In these experiments physical association between a LexA-Pnr fusion protein and Ush fused to the B42 activation domain was detected by activation of a Leu2 reporter gene that contains upstream LexA-binding sites (Gyuris et al. 1993; Finley and Brent 1994; the wild type as well as the different mutated versions of Pnr do not activate transcription in yeast). As shown in Figure 6, Ush interacts with the LexA-Pnr', LexA-Pnr ${ }^{\mathrm{VX}}$, and LexA-Pnr ${ }^{\vee \times 4}$ fusion proteins, which all carry a wild-type DBD. In contrast, this interaction is lost with fusion proteins constructed with the DBD carrying the point mutations characteristic of the $\mathrm{pnr}^{\mathrm{D}}$ class of dominant alleles (LexA-Pnr ${ }^{D 1}$ to LexA-Pnr ${ }^{D 4}$ ). The specificity of the interaction is further underlined by the use of the unrelated LexA-Bicoïd fusion protein (Fig. 6).

To ascertain that Ush associates specifically with the DBD of Pnr, we performed GST fusion protein selection experiments with protein extracts from Cos cells cotransfected with vectors for the tagged $U$ sh protein and a fusion between GST and the complete Pnr-DBD by itself (GST-Pnr-DBD; see M aterials and M ethods). As shown in Figure 5, Ush associates specifically with the GSTPnr-DBD fusion protein but not with GST al one. Therefore, it interacts with the isolated Pnr-DBD. Furthermore, U sh also associates with a fusion protein between GST and the highly related DBD from the CGATA-1 transcription factor (GST-GATA-1-DBD; Fig. 5D, Iane 3). Over a stretch of 115 amino acids in Pnr and 116 in cGATA-1, 85 are identical (74\% identity).

To define further the domain of Pnr required for association with Ush, we expressed fusion proteins bearing either the amino or the carboxy-terminal zinc finger sequences fused to GST (fusion proteins GST-Pnr-ZnN 


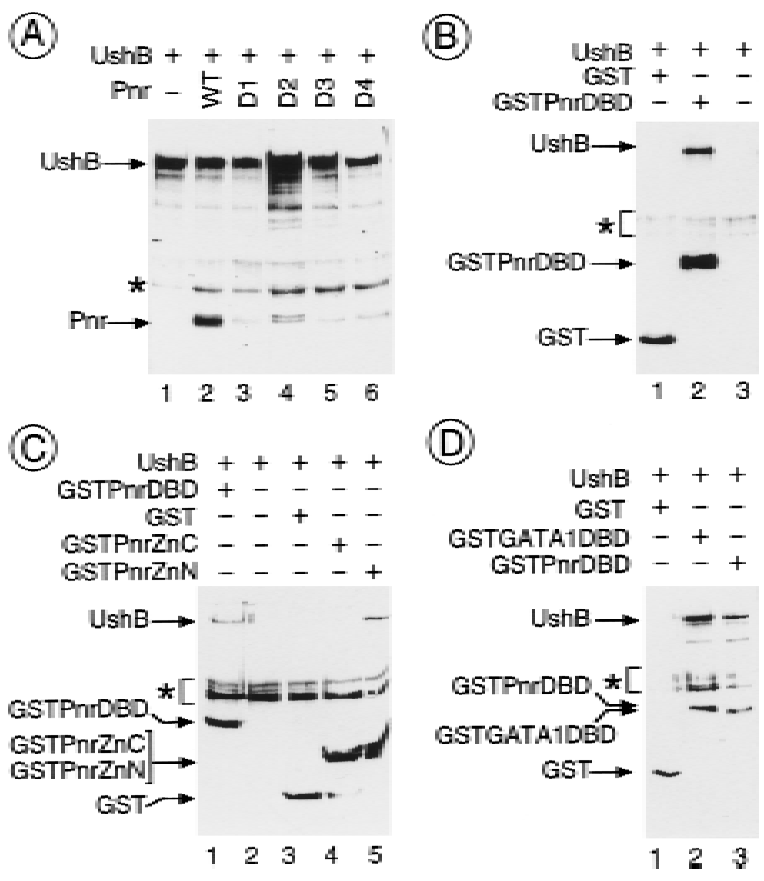

Figure 5. Direct in vitro protein-protein interactions between Ush and Pnr. Cos cells were cotransfected with $5 \mu \mathrm{g}$ of each expression vector, as denoted above each panel, and the corresponding protein extracts were either immunoprecipitated (A) with the B10 monoclonal antibody raised against the $B$ epitope of the estrogen receptor inserted into the Ush open reading frame or incubated with glutathione-agarose (B-D). Selected proteins were anal yzed by Western blot. U sh-B is detected with the B10 antibody, Pnr and Pnr ${ }^{\mathrm{D}}$ with the specific monoclonal antibody 2B8, and the GST fusion proteins with the GST-specific monoclonal antibody 1D10. (A) In vitro interactions between Ush and wild-type Pnr as well as Pnr ${ }^{\mathrm{D}}$. Arrows indicate the Ush-B (lanes 1-6) and Pnr (lanes 2-6: wild-type Pnr, Pnr ${ }^{\mathrm{D} 1}$, $\mathrm{Pnr}^{\mathrm{D} 2}, \mathrm{Pnr}^{\mathrm{D} 3}$ and $\mathrm{Pnr}^{\mathrm{D} 4}$, respectively). The asterisk $\left(^{*}\right)$ denotes the location of the immunoglobulin heavy chain. (B) In vitro interactions between Ush and the isolated Pnr-DBD. Arrows indicate U sh-B (lane 2), GST (lane 1), and the fusion GST-PnrDBD (lane 2). The asterisk denotes artifactual bands. (C) In vitro interactions between $U$ sh and Pnr isolated zinc fingers. Arrows indicate U sh-B (lanes 1,5), GST al one (lane 3), the fusion GST Pnr-DBD (lane 1), and the fusion proteins GST-Pnr-ZnC (lane 4) and GST-Pnr-ZnN (lane 5). The asterisk denotes artifactual bands. (D) In vitro interactions between Ush and the cGATA1-DBD. Arrows indicate U sh-B (lanes 2,3), GST al one (lane 1), and the fusion proteins GST -Pnr-DBD (lane 2) and GST -GATA1-DBD (lane 3). The asterisk denotes artifactual bands.

and GST-Pnr-ZnC, respectively; Fig. 5C). Ush does not bind the GST-Pnr-ZnC fusion protein but does associate with the amino-terminal zinc finger fusion protein as efficiently as with the complete DBD (Fig. 5C, lanes 1,4,5). It is noteworthy that the $\mathrm{Pnr}^{\mathrm{D}}$ proteins are all characterized by point mutations in the amino-terminal zinc finger and that the amino acids that are mutated are conserved perfectly within the GATA family of transcription factors.
Overexpression of wild-type and mutant pnr proteins in transgenic flies regulates ac-sc expression through the dorsocentral enhancer

We have shown that wild-type Pnr, as well as the $\mathrm{pnr}^{\mathrm{D}}$ proteins that bear lesions in the amino-terminal zinc finger, stimulate transcription from the heterol ogous $\alpha$-globin promoter. In contrast the $\mathrm{Pnr}^{\mathrm{V} \times 1 / 4}$ proteins, which carry deletions of the amphi pathic $\alpha$-helices in the carboxyl region of Pnr, do not activate in this assay. Heterozygous flies mutant for $\mathrm{pnr}^{\mathrm{D}}$ differentiate extra dorsocentral bristles resulting from overexpression of ac-sc, whereas heterozygous flies mutant for $\mathrm{pnr}^{\mathrm{V} \times 1 / 4}$ differentiate fewer dorsocentral bristles attributable to decreased ac-sc expression (Ramain et al. 1993). We have now analyzed the effects of overexpression of these three pnr proteins on the devel opment of the dorsocentral bristles and on the activity of a lacZ reporter gene whose expression is under the control of specific enhancer sequences that drive ac-sc expression very strongly at the dorsocentral site (Gomez-Skarmeta et al. 1995). We made use of the GAL4/UAS system (Brand and Perrimon 1993), using as a driver the $\mathrm{pnr}^{\mathrm{MD} 237}$ strain that carries a GAL4-containing transposon inserted at the pnr locus. This gives an expression pattern indistinguishable from that of pnr and does not display a mutant bristle pattern on the thorax (Calleja et al. 1996; Heitzler et al. 1996).

Overexpression of either the wild-type or the $\mathrm{Pnr}^{\mathrm{D} 4}$ protein leads to an excess of dorsocentral bristles ( $T$ able 1; Fig. 7, A and C, respectively). The wild-type protein causes a modest effect of one or two additional bristles per hemithorax in $15 \%-25 \%$ of the transgenic flies. $\mathrm{Pnr}^{\mathrm{D} 4}$, however, causes a dramatic increase of 4-10 additional bristles per hemithorax in all of the flies, and furthermore, viability of these animals is strongly reduced (Table 1). In contrast, overexpression of $\mathrm{Pnr}^{\mathrm{V} \times 4}$ leads to a loss of dorsocentral bristles (Table 1; Fig. 7B).

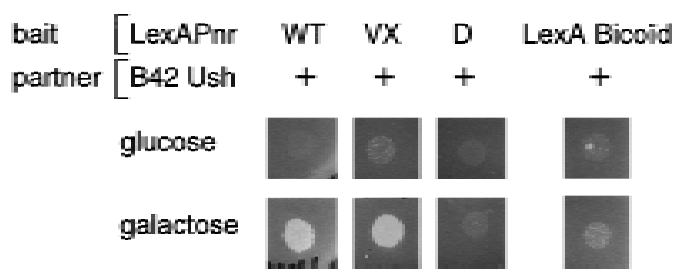

Figure 6. In vivo interactions between Ush and Pnr in yeast. Yeast transformed by an expression vector encoding the bait LexA-Pnr (the different forms of the protein were wild-type Pnr, $\mathrm{Pnr}^{\mathrm{V} \times 1}$, or $\mathrm{Pnr}^{\mathrm{D} 1}$ ) and an expression vector encoding the partner B42-Ush were plated on a medium lacking histidine, trytophane, and leucine, with either glucose, where the expression of B42-U sh is repressed, or with gal actose, where expression of the partner is induced. The results were the same for LexA-Pnr ${ }^{V X 4}$, LexA-Pnr ${ }^{\mathrm{2}}$, LexA-Pnr ${ }^{\mathrm{D} 3}$, and LexA-Pnr ${ }^{\mathrm{D} 4}$ (data not shown). The specificity of the interaction is further demonstrated by the use of the LexA-Bicoid unrelated fusion protein. Levels of LexA-Pnr fusion proteins in the different strains were monitored by Western blot analysis using the Pnr-specific monoclonal antibody 2B8 (data not shown). 
Table 1. Number of dorsocentral bristles present in flies of different genotypes following overexpression of Pnr, Pnr ${ }^{\mathrm{D} 4}, \mathrm{Pnr} \mathrm{Vx}^{\mathrm{N}}$ or Ush

\begin{tabular}{|c|c|c|c|c|}
\hline Construct & Genetic background & $\begin{array}{c}\text { Percent mutant } \\
\text { heminota }\end{array}$ & $\begin{array}{c}\text { A verage no. } \\
\text { dorsocentral } \\
\text { bristles/heminota }\end{array}$ & No. \\
\hline None & $\mathrm{pnr}^{\mathrm{MD} 237} /+$ & 0 & 2.0 & 50 \\
\hline UAS:Pnr ${ }^{W T^{a}}$ & $\mathrm{pnr}^{\mathrm{MD} 237} /+$ & 19 & $2.2 \pm 0.01$ & 618 \\
\hline UAS:Pnr'WT & $\mathrm{ush}^{-} /+; \mathrm{pnr}^{\mathrm{MD} 237} /+$ & 83 & $3.48 \pm 0.07$ & 178 \\
\hline None & $\mathrm{ush}^{-1}+; \mathrm{pnr}^{\mathrm{MD} 237} /+$ & 0 & 2.0 & 86 \\
\hline UAS: $P n r^{\vee \times 4^{a}}$ & $\mathrm{pnr}^{\mathrm{MD} 237} /+$ & 99 & $0.29 \pm 0.01$ & 618 \\
\hline UAS: $P n r^{D 4^{a}}$ & $\mathrm{pnr}^{\mathrm{MD} 237} /+$ & 100 & $7.35 \pm 0.2$ & $56^{\mathrm{b}}$ \\
\hline UAS: Ush'14AII & pnrMD237/+ & 100 & 0 & $8^{b}$ \\
\hline UAS: Ush ${ }^{14 A I I}$ & $\mathrm{pnr}^{\mathrm{D} 1} / \mathrm{pnr} r^{\mathrm{MD} 237}$ & 100 & $3.3 \pm 0.6$ & 22 \\
\hline N one & $\mathrm{pnr}^{\mathrm{D} 1} / \mathrm{pnr} r^{\mathrm{MD} 237}$ & 100 & $7.6 \pm 0.6$ & 25 \\
\hline None & $\mathrm{pnr} r^{\mathrm{D} 1} /+$ & 100 & $2.7 \pm 0.16$ & 26 \\
\hline
\end{tabular}

Each transgenic strain carried one transposon.

${ }^{\text {aA }}$ verages of three independent insertion lines are given.

'T hese animals showed strongly reduced viability.

These observations correlate perfectly with ac-lacZ reporter gene expression at the dorsocentral site (GomezSkarmeta et al. 1995; Fig. 8A). Staining is weakly reinforced by overexpression of Pnr (Fig. 8B), but becomes very intense after overexpression of $\mathrm{Pnr}^{\mathrm{D} 4}$ and completely covers the dorsal-most region of the thoracic disc delimited by the prescutum, the postnotum, and the dorsocentral area (Fig. 8D). This is consistent with other observations showing that in $\mathrm{pnr}^{\mathrm{D} 4}$ mutants expression of this reporter is al so increased (data not shown). This corresponds to the domain where pnr and ush are expressed simultaneously in the wild type. In contrast, overexpression of $\mathrm{Pnr}^{\mathrm{V} \times 4}$ leads to a strong reduction of lacZ staining in the dorsocentral area (Fig. 8C). Thus, there is a correlation between the effects of the three protein forms on ac-sc expression and bristle develop- ment and their transcriptional activity on the globin promoter in the transfection experiments.

Overexpression of the ush protein in transgenic flies reduces ac-sc expression in the wild-type but not in the $\mathrm{pnr}^{\mathrm{D}}$ mutants

Overexpression of Pnr in a genetic background associated with a reduced amount of the Ush product [Df(3R)ush ${ }^{\text {Rev18 }}$ ush $^{-} /+$] leads to an increase in the fre quency of phenotypically mutant flies, as well as an increase in the number of extra dorsocentral bristles (Table 1; Fig. 7D). Because Ush regulates Pnr negatively in the assay using the globin promoter, this suggests an antagonistic role of Ush on Pnr activity during bristle patterning. To verify this we looked at the effects of overexpres-

Figure 7. Effect of overexpression of the different pnr proteins on development of the dorsocentral bristles. Homozygous transgenic strains carrying a UAS-Pnr transposon were crossed with pnr $^{\mathrm{MD} 237} /$ TM6b; Tb flies and the $\mathrm{Tb}^{+}$flies carrying the pnr ${ }^{\mathrm{MD} 237}$ driver were analyzed. Arrows denote additional dorsocentral bristles in $A, C$, and D, whereas asterisks (*) indicate the positions of the missing bristles in $B$. (A) Strain UAS-Pnr'. (B) Strain UAS$\mathrm{Pnr}^{\vee \times 4}$. (C) The transgenic strain UAS$\mathrm{Pnr}^{\mathrm{D} 4}$. (D) Strain UAS-Pnr ${ }^{+}$(same as in (A) in the presence of a reduced amount of $U$ sh [Df(3R) ush ${ }^{\text {Rev18, }}$ ush $^{-1+}$ ].
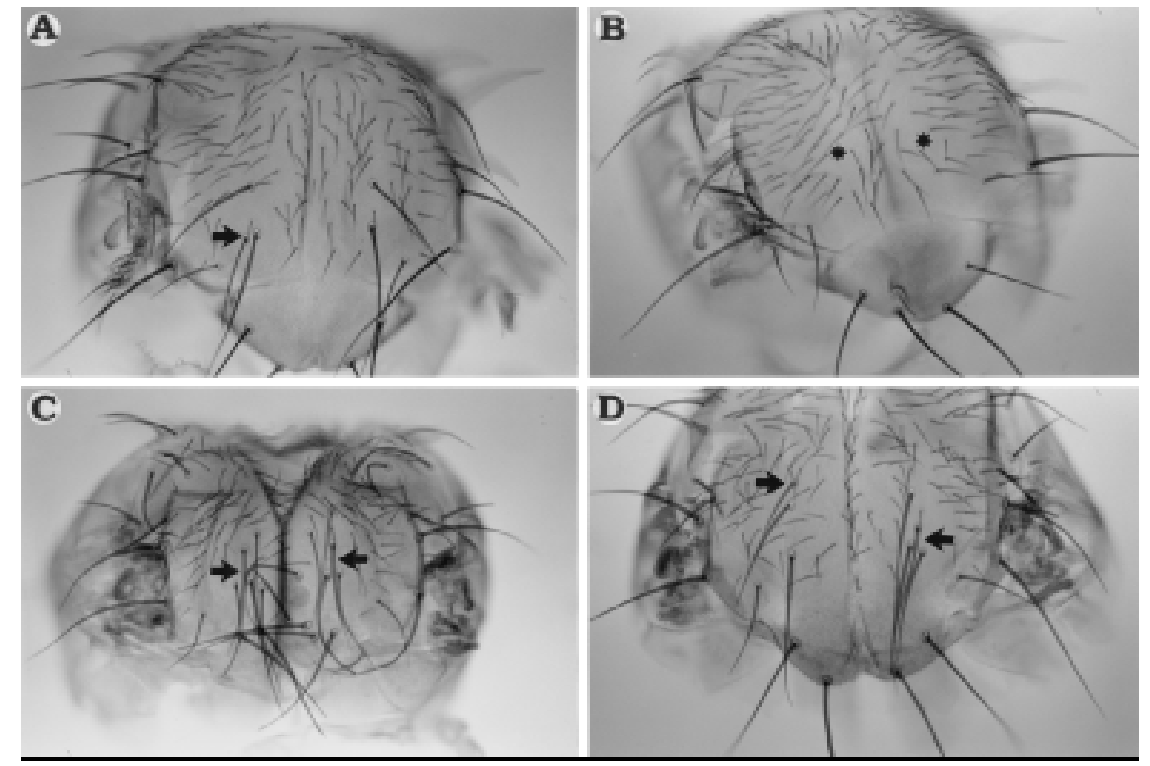
Negative regulation of Pannier by u-shaped
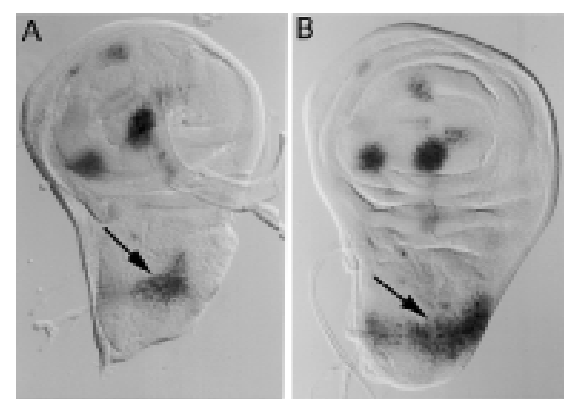

E

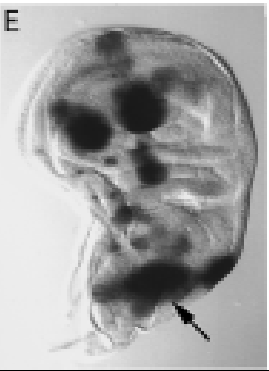

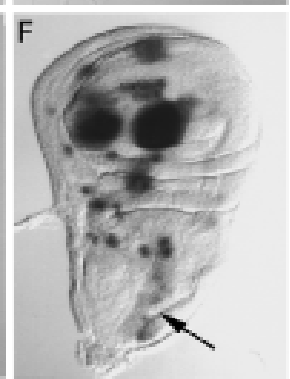

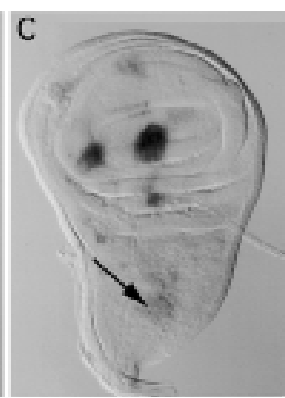

G
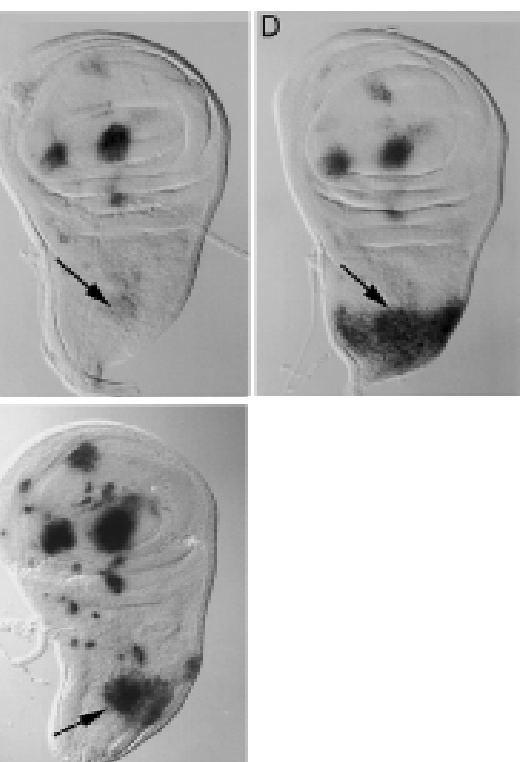

Figure 8. The effects of overexpression of the different forms of pnr proteins and ush on the lacZ reporter whose expression is driven by the dorsocentral-specific enhancer sequences (Gomez-Skarmeta et al. 1995). Arrows denote lacZ expression in the dorsocentral region of the thoracic disc. (A-D) Homozygous transgenic flies carrying a UAS-Pnr transposon were crossed with DC-enhancersc-lacZ; pnr ${ }^{\mathrm{MD} 237} / \mathrm{TM} 6 \mathrm{~B}$ Tb flies, and the resulting larvae of the appropriate genotype (see below) were dissected and the imaginal discs stained for $\beta$-galactosidase activity. In each case, the reaction was left for $3 \mathrm{hr}$ at $22^{\circ} \mathrm{C}$; longer reaction times led to background staining, which prevents comparison between the different pnr proteins. (E-G) Overexpression of ush leads to the reduction of ac-sc expression in $\mathrm{pnr}^{\mathrm{MD} 237} /+(\mathrm{F})$ but not $\mathrm{pnr}^{\mathrm{D}} / \mathrm{pnr}^{\mathrm{MD} 237}$ mutants $(\mathrm{G})$. In E-G the reaction was left for $6 \mathrm{hr}$ at $37^{\circ} \mathrm{C}$ to emphasize the fact that overexpression of Ush leads to complete repression of the lac $Z$ reporter. (A) Genotype (U AS-Pnr'WT/lacZ; TM6B, Tb/ +); (B) genotype (UAS-PnrWT/lacZ; pnr ${ }^{\mathrm{MD} 237} /$ +); (C) genotype (U AS-Pnr ${ }^{\mathrm{VX} 4} /$ lacZ; pnr ${ }^{\mathrm{MD} 237}$ / +); (D) genotype (U AS-Pnr $\left.{ }^{\mathrm{D}} / \mathrm{lacZ} ; \mathrm{pnr}^{\mathrm{MD} 237} / \mathrm{H}\right)$; (E) genotype (UAS-U sh/lacZ; TM6B, Tb/ H); (F) genotype (UAS-U sh/lacZ; pnr ${ }^{\mathrm{MD} 237 /}$ TM6B, Tb); (G) genotype (U AS-U sh/lacZ; $\left.\mathrm{pnr}^{\mathrm{MD} 237} / \mathrm{pnr}^{\mathrm{D} 1}\right)$.

sion of Ush in flies expressing the mutant pnr protein $\mathrm{Pnr}^{\mathrm{D}}$, which displays in vitro a weaker association with Ush. Whereas in wild-type flies overexpression of Ush results in a loss of ac-sc reporter gene expression (Fig. $8 \mathrm{~F}$ ), in $\mathrm{pnr}^{\mathrm{D}}$ heterozygotes it has a milder effect and staining still remains fairly strong (Fig. 8G). This suggests that in the thoracic epithelium Ush antagonizes the effects of Pnr, leading to ac-sc expression and bristle development.

\section{Discussion}

Pnr binds to the consensus GATAAG sequence and activates transcription

The DBD s of members of the GATA family of transcription factors are highly rel ated and here we demonstrate that Pnr is a transcription factor, it binds DNA, and can activate transcription of the GATA-1 target promoter of the $\alpha$-globin gene in transfected CEFs. A binding site enrichment protocol has been used to define a consensus for the Pnr DNA-binding domain sequence and the optimal site appears to be a GATAAG motif. This is similar to the consensus proposed by Plumb et al . (1989) and close to the WGATAR sequence derived previously by an in vivo analysis (Yamamoto et al. 1990). M ost GATA factors, like Pnr, carry two zinc fingers. In an extensive in vitro study, Whyatt et al. (1993) described two different classes of DN A-binding sites for GATA-1, the first requiring only the carboxy-terminal zinc finger to bind the motif GAT (A/T), the second requiring both zinc finger motifs to bind the (T/C)AAG sequence. Thus, it is possible that the consensus sequence that we have se- lected, GATAAG, may correspond to two overlapping recognition sequences where GATA is recognized by the carboxy-terminal zinc finger and TAAG by the complete DBD.

Our results suggest that in the case of Pnr, the aminoterminal zinc finger is not required for binding to the consensus sequence present in the globin promoter, or indeed activation, as the mutant $\mathrm{Pnr}^{\mathrm{D}}$ proteins bearing lesions in the amino-terminal zinc finger are capable of transcriptional activation in a transient expression assay. It is noteworthy that, unlike the vertebrate proteins, the yeast GATA proteins bear only a single zinc finger, with a similar sequence to the carboxy-terminal zinc finger of vertebrate GATA factors. This suggests that, in the case of Pnr and the vertebrate proteins, the carboxy-terminal zinc finger is the crucial determinant for DNA binding. Indeed deletions within this region, or point mutations of the conserved cysteine residues, abolish binding of the protein. Furthermore, binding of the vertebrate proteins to the GAT(A/T) sequence correlates with transcriptional activation (deBoer et al. 1988; Mignotte et al . 1989a, b; N icolis et al. 1991; T sai et al. 1991; Simon et al. 1992; Philipsen et al. 1993) or with indirect transcriptional activity through the displacement of a repressor (Rahuel et al. 1992).

The amino-terminal zinc finger of GATA-1 does not bind DNA by itself, and may be involved in the discrimination between different GATA sites present in the control regions of erythrocyte genes (Y ang and Evans 1992). There is recent evidence, however, that the amino-terminal zinc finger of GATA-2 and GATA-3 can bind a GATC sequence (Pedone et al. 1997). Binding is dependent on the presence of two basic regions on either side 
of the finger. One of these regions, the amino-terminal, is missing in GATA-1, which is unable to bind in the assay used by these investigators. This domain is also absent in Pnr.

U sh associates with the amino-terminal zinc finger of Pnr and regulates transcriptional activity negatively

The DNA-binding domain of GATA factors is also involved in dimerization processes. Thus, the GATA proteins homodimerize by means of either the amino- or carboxy-terminal zinc fingers (Crossley et al. 1995). GATA-1 has al so been shown to heterodimerize with the transcription factors Spl and EKLF, which belong to the Krüppel family and carry $\mathrm{C}_{2} \mathrm{H}_{2}$ zinc finger motifs (M iller and Bieker 1993; Merika and Orkin 1995). This associati on requires the GATA carboxy-terminal zinc finger and the interaction leads to synergistic transcriptional activity. In contrast, our study illustrates a different mode of regulation of GATA function by means of heterodimerization through the amino-terminal zinc finger. We have shown that the function of Pnr is regulated by association with $\mathrm{U}$ sh, a large protein bearing both $\mathrm{C}_{2} \mathrm{HC}$ and $\mathrm{C}_{2} \mathrm{H}_{2}$ zinc fingers (Cubadda et al ., this issue). The physical association requi res a wild-type GATA-DBD. Indeed, when the DBD carries a single point mutation in the amino-terminal zinc finger, as in the $\mathrm{Pnr}^{\mathrm{D}}$ proteins, association of the two proteins is reduced drastically. This suggests that the amino acids that have been lost either interact directly with U sh or are crucial determinants for the highly ordered structure of the zinc finger required for heterodimerization. All of the mutations involve amino acids that are conserved completely among the members of the GATA family, and furthermore $p n r^{D 1}$ and $\mathrm{pnr}^{\mathrm{D} 3}$ have substitutions of the conserved cysteine residues that probably maintain the structural integrity of the zinc finger (Ramain et al. 1993). Thus, heterodimerization involving the amino-terminal zinc finger of a GATA factor uncovers a novel mode of regulation of GATA function during development.

In the transient assay that we have used, Ush reduces the transcriptional activity of Pnr by heterodimerization. To determine whether the DNA-binding properties of Pnr are disrupted, we performed EMSA with protein extracts made from transfected Cos cells and we were unable to detect either ternary complex on the globin probe or reduced binding of Pnr in presence of U sh (data not shown). Further studies are required to resolve this point. Ush also interacts with CGATA-1, raising the possibility of the existence of vertebrate proteins homologous to Ush. Indeed, recently a zinc finger protein, FOG (friend of GATA-1), structurally similar but not homologous to $U$ sh, has been recovered from a yeast two-hybrid screen using the vertebrate GATA-1 as bait (T sang et al . 1997). Like Ush, this protein heterodimerizes with GATA-1 through the amino-terminal zinc finger. In contrast to Ush, however, FOG has a synergistic effect on the activation of transcription by GATA-1. These observations suggest the possible existence of a family of simi-
Iar proteins that modulate the transcriptional activity of the different GATA factors.

Antagonistic activities of Pnr and $U$ sh during bristle patterning

Mutants of pnr affect the level of ac-sc expression in the imaginal discs of the fly and al ter the bristle pattern. The $\mathrm{pnr}^{\mathrm{D}}$ heterozygotes are associated with an over-expression of ac-sc in the dorsocentral area and the formation of additional bristles at this site (Ramain et al. 1993). On the other hand, pnr ${ }^{\vee \times 1 / 4}$ heterozygotes are associated with a loss of ac-sc expression and a loss of dorsocentral bristles. In an earlier study we interpreted the $\mathrm{pnr}^{\mathrm{D}}$ mutants as representing a loss of pnr function, thinking that the lesions in the amino-terminal zinc finger would abolish activity of the DBD. In this study, however, we have shown that the mutated $\mathrm{Pnr}^{\mathrm{D}}$ proteins activate transcription as efficiently as the wild type in a transient expression assay involving the heterologous $\alpha$-globin promoter. In contrast, the $\mathrm{Pnr}^{\mathrm{V} \times 1 / 4}$ proteins are unable to stimulate this promoter. Therefore, it is possible that in vivo Pnr acts as a transcriptional activator of ac and sc. Overexpression of Pnr or $\mathrm{Pnr}^{\mathrm{D}}$ using the GAL4-UAS system also leads to the formation of additional bristles. We have shown that the effects of Pnr appear to be mediated by the specific enhancer sequences required for expression of ac-sc at the dorsocentral site (GomezSkarmeta et al. 1995). It remains to be seen whether Pnr acts directly on these sequences or indirectly through intermediate genes.

The direct association of the Pnr and U sh proteins that we describe here could be the molecular basis underlying the similar phenotypes of $\mathrm{pnr}^{\mathrm{D}}$ gain-of-function mutants and ush loss-of-function mutants, as well as the genetic interactions observed between mutant alleles of these two genes. Ush behaves genetically as a repressor of ac and Sc; hypomorphic alleles accumulate higher levels of ac-sc and form additional bristles, whereas overexpression of U sh leads to a loss of bristles (Cubadda et al., this issue). $\mathrm{Pnr}^{\mathrm{D}}$ heterodimerizes only poorly with Ush, which would mean that in vivo it would stimulate transcription more strongly and over a broader territory. Overexpression of $\mathrm{Pnr}^{\mathrm{D} 4}$ causes a dramatic increase in the levels of ac-sc-lacZ, as does over-expression of wildtype Pnr in animals heterozygous for ush. The phenotype of heterozygous $\mathrm{pnr}^{\mathrm{D}} /+$ flies is enhanced when the dosage of ush $^{+}$is reduced and suppressed when it is increased (Heitzler 1993; Cubadda et al ., this issue). Other alleles do not display phenotypic changes in the presence of varying amounts of Ush.

One explanation for the specificity of this interaction could be attributable to the fact that the two genes are expressed in overlapping, but not precisely coincident, areas of the thorax; the pnr domain is slightly more extensive than that of ush (Ramain et al. 1993; Cubadda et al., this issue). Furthermore, the dorsocentral bristle precursors arise in a domain of high pnr expression but low ush expression (Cubadda et al., this issue). Down-regulation of Pnr by Ush is restricted presumably to the do- 
main in which both genes are expressed. The $\mathrm{Pnr}^{\mathrm{D}}$ protein would be more or less resistant to U sh, therefore, in $\mathrm{pnr}^{\mathrm{D}}$ flies Ac-Sc levels would increase and cause the appearance of ectopic bristles in the ush expression domain in addition to the ones that devel op in the normal $\left(\mathrm{Pnr}^{+} \mathrm{Ush}\right)$ territory. On the other hand, the phenotype of alleles that cause a loss of pnr expression or function, such as pnr ${ }^{\vee \times 1 / 4}$, would be attributable to a loss of Pnr activity in the nonoverlapping domain of high pnr but low ush expression. Pnr in this area would not be regulated by Ush anyway.

Consistent with these observations, overexpression of Ush, which in wild-type flies leads to a loss of ac-sc expression, has only a mild effect on the levels of ac-sclacZ in pnr ${ }^{D}$ mutant flies. Thus, Pnr and Ush appear to have antagonistic effects on the expression of ac-sc. Thus, it is possible that ac-sc levels are higher in areas of lower ush expression and that Pnr and U sh contribute to the precise positioning of dorsocentral and perhaps other bristles.

\section{Materials and methods}

All recombinant DNA work was performed according to standard procedures (Sambrook et al . 1989). Details concerning plasmid constructions, which were all verified by sequence analysis performed with an Applied Biosystems automated DNA sequencer, are available upon request. N ucl eotides and amino acids are numbered with reference to our previous report (Ramain et al. 1993).

\section{Plasmid constructions}

A 2900-bp HindlII-EcoRI DNA fragment containing the complete wild-type open reading frame of Pnr and isolated from our original PN B40 clone (Ramain et al. 1993) was subcloned into pBluescript SK+ to give $\mathrm{SK}^{+} \mathrm{Pnr} r^{+}$. The point mutations corresponding to the different $P n r^{D}$ proteins ( $D 1$ to $D 4$ ), as well as the deletions corresponding to the $\mathrm{Pnr}^{\mathrm{VX}}$ proteins ( $\mathrm{VX} 1$ and $\mathrm{VX} 4$ ) were introduced by site-directed mutagenesis using the $\mathrm{SK}^{+} \mathrm{Pnr}^{+}$ single-strand DNA as a template and the following oligonucleotides: D1, 5'-AGGGACGCGAGTACGTCAATTGCGG-3'; D2, 5'-GGGATGGAACCGAACACTATCTGTG-3'; D3, 5'-GGACACTATCTGAGCAACGCCTGCG-3'; D4, 5'-GGCGAGGGACGCAAGTGCGTCAATT-3'; VXI, 5'-CCTTCACGGAGCTCTACACGCCCG-3'; VX4, 5'-CAGGAACGCAGCAGTTCCGGCGGA-3'

For transfection experiments, the different HindlII-N otl fragments of the pBluescript SK+derivatives were inserted into the pXJ vector (Xiao et al. 1991), whose expression is driven in transfected cells by the cytomegal ovirus promoter sequences. Using M 136 as an internal start codon, the truncated Pnr protein is produced with the expression vector $\mathrm{pXJ}$ containing the Sphl(870)-Notl DN A fragment. A molecular mass of $45 \mathrm{kD}$, as expected, was found by Western blot analysis with the help of the monoclonal antibody $2 \mathrm{~B} 8$, produced in mouse and raised against the peptide TTQQQHQQHGHSMTSSSGQA (amino acids 378-397). The expression vector carrying the Pnr-TEF-1 chimera was constructed by inserting the HindlII-BamHI DNA fragment containing the Pnr-DBD from $\mathrm{SK}^{+} \mathrm{Pnr}^{+}$and the BamHI fragment from pXJ (Gal4-TEF-1; Xiao et al. 1991) containing the TEF-1 activation domain (amino acids 167-426) into pXJ. Similarly, the expression vector carrying the Gal4-Pnr chimera was constructed by exchanging the BamHI fragment from $\mathrm{pXJ}$ (Gal4TEF-1) containing the TEF-1 activation domain with the BamHI fragment containing the carboxyl terminus of Pnr isolated from $\mathrm{SK}^{+} \mathrm{Pnr} r^{+}$.

The expression vectors used in the yeast interaction assays were constructed in the plasmid pEG202, which encodes the bait fused to the LexA-DBD, and in the plasmid pJG4-5, where the putative partner is fused to the bacterial B42 activation domain that is efficient in yeast. A repaired Ndel-Notl fragment containing the complete Pnr open reading frame was isolated from a pBluescript SK $+\mathrm{Pnr}^{+}$derivative where a $\mathrm{N}$ del restriction site was created on the ATG by site-directed mutagenesis using the oligonucleotide (5'-CGGCCATAAATCCATATGGGCATCTTACTG-3'; Ndel, underlined) and inserted into the repaired EcoRI-Notl cloning sites from pEG202 to give pEG202Pnr ${ }^{+}$. The pEG202Pnr ${ }^{\mathrm{D} 1}$ to $\mathrm{pEG} 20 \mathrm{Pnr} \mathrm{D}^{\mathrm{D}}$ expression vectors were constructed by exchange of the wild-type Sall fragment from $\mathrm{pEG} 202 \mathrm{Pnr}^{+}$with the mutated Sall fragments from pXJPnr ${ }^{D 1}$ to $p X J P n r^{D 4}$. The pEG202Pnr ${ }^{\vee X 1}$ and pEG202Pnr $r^{\mathrm{VX} 4}$ expression vectors were constructed by exchange of the BamHI-N otl fragment from pEG202Pnr ${ }^{+}$with the BamHI-N otl fragments from $\mathrm{SK}^{+} \mathrm{Pnr}{ }^{\vee \times 1 / V \times 4}$. pJG4-5 was modified by insertion into the EcoRI and Xhol cloning sites of the complementary ol igonucleotides (5'-AATTCGCTAGCTAAC-3' and 5'-TCGAGTTAGCTAGCG-3') creating an Xbal cloning site (underlined). A 4-kb Xbal fragment isolated from SK+Ush and encompassing the complete open reading frame was then inserted into the Xbal site of the pJG4-5 derivative.

The selection of GST fusion proteins was performed with extracts made from Cos cells transfected with $\mathrm{pBC}$ derivatives (Stratagene, La Jolla, CA; Chatton et al. 1995) allowing the expression of GST fusion proteins in a cultured cell line. Thus, the complete Pnr-DBD as well as the isolated zinc fingers were generated by site-directed mutagenesis using as a template the $\mathrm{SK}^{+} \mathrm{Pnr}{ }^{+}$single-stranded DNA and the following oligonucleotides carrying an $N$ hel restriction site (underlined): $5^{\prime}$-GGAAGGATTCGCTAGCGCGCATGCAC-3' and 5'-CACTGGATCCGCTAGCGGCTCCACTT-3' (Pnr-DBD); 5'-GGAAGGATTCGCTAGCGCGCATGCAC-3' and 5'-TGTGAGTGCAGCTAGCACTGCCACCC-3' (Pnr-ZnN); 5'-TGTGAGTGCAGCTA $\overline{\text { GC }}$ ACTGCCACCC-3' and 5'-CACTGGATCCGCTAGCGGCTCCACTT-3' (Pnr-ZnC).

The $\mathrm{SK}^{+}$derivatives were then restricted by $\mathrm{Nhel}$ and the DNA fragments encompassing the complete DBD as well as the isol ated Pnr zinc fingers were inserted into the $\mathrm{N}$ hel cloning site of $\mathrm{pBC}$ to give $\mathrm{pBC}-\mathrm{Pnr}-\mathrm{DBD}, \mathrm{pBC}-\mathrm{Pnr}-\mathrm{ZnN}$, and $\mathrm{pBC}-\mathrm{Pnr}-$ $\mathrm{ZnC}$, allowing the production of GST fusion proteins containing the amino acids alanine 124-serine 292 (GST-Pnr-DBD), alanine 124-alanine 216 (GST-Pnr-ZnN), and threonine 217serine 292 (GST-Pnr-ZnC), respectively. The pBC-GATA-1DBD expression vector was generated by insertion of a 421-bp Smal fragment containing the cGATA-1-DBD sequences (glycine 85-proline 224) and isolated from pRSV20.2 (Evans and Felsenfeld 1991) into the $\mathrm{N}$ del repaired cloning site of pBC.

\section{PCR-assisted DNA-binding site selection}

The Nhel fragment containing the Pnr-DBD sequences was inserted into the pGEX-3X vector, allowing the production of GST fusion proteins in Escherichia coli. The resulting plasmid was introduced by transformation into BL21 (DE3) lysS competent cells. Five hundred milliliters of Luria broth plus $100 \mu \mathrm{g} / \mathrm{ml}$ of ampicillin were innoculated with a $10-\mathrm{ml}$ saturated overnight culture grown at $30^{\circ} \mathrm{C}$ and incubated at the same temperature until it reached an OD of 0.6-0.7 at $600 \mathrm{~nm}$. Isopropyl- $\beta$-thiogal actopyranoside (IPTG) was added to a final concentration of 
$1 \mathrm{~mm}$ and the culture was further incubated for $2 \mathrm{hr}$. Cells were harvested by centrifugation, washed in cold PBS, and lysed by sonication in $1 \times$ PBS with $1 \%$ Triton X-100. The bacteria debris was el iminated by centrifugation and the supernatant applied to a glutathione-agarose (Pharmacia) column equilibrated with $1 \times$ PBS, 1\% Triton X-100. After extensive, successive washes with $1 \times$ PBS, $1 \times$ PBS, $1 \mathrm{M} \mathrm{N} \mathrm{aCl}, 1 \%$ Triton $X-100$, and the binding buffer [50 mm Tris- $\mathrm{HCl}(\mathrm{pH} 7.8), 50 \mathrm{~mm} \mathrm{KCl}, 10 \%$ glycerol, 1 mм DTT, $100 \mu \mathrm{m} \mathrm{ZnSO}_{4}, 100 \mu \mathrm{g} / \mathrm{ml}$ poly[d(I-C)], the labeled degenerated oligonucleotides were loaded on the column. The random sequence oligonucleotide: [5'-CTGGATCCTAGATGTCCCTG (N ) 10 AGGCTCAAAGCTGAATTCCT-3'] was rendered double stranded with the Klenow polymerase by primed synthesis using the primer (PL192: 5'-AGGAATTCAGCTTTGAGCCT-3') labeled with [ $\gamma^{-32}$ P]ATP. After purification on an acrylamide gel, electroelution, and precipitation, the probe was resuspended in binding buffer. After extensive washes with the binding buffer plus 1\% Triton X-100, the selected oligonucleotides were eluted stepwise with the binding buffer containing increasing concentrations of $\mathrm{KCl}$, and the oligonucleotides eluted at a 500-mM KCl concentration were precipitated, recovered by centrifugation, and resuspended in water before PCR amplification with the PL192 and QK61(5'-CTGGATCCTAGATGTCCCTG-3') primers labeled with $\left[\gamma^{-32}{ }^{32}\right.$ PATP. The amplified product, purified on an acrylamide gel, was applied again to the column and after four cycles of enrichment the selected oligonucleotides were restricted by BamHI and EcoRI and subcloned into pBluescript SK + for further sequence analysis. They were also used as a template in an EM SA. The GST and GSTPnr-DBD fusion proteins were purified with glutathione-agarose according to the recommendations of the manufacturer and the protein concentration was estimated by the Bradford assay and Coomassie staining after gel el ectrophoresis. For the EMSA, the purified proteins were incubated with the pool of selected oligonucleotides $\left(50 \times 10^{3} \mathrm{cpm}\right)$ in the binding buffer $[20 \mathrm{~mm}$ HEPES (pH 7.9), $1 \mathrm{~mm} \mathrm{DTT,} 20 \%$ glycerol, $100 \mathrm{~mm} \mathrm{KCl]} \mathrm{during}$ $20 \mathrm{~min}$ at room temperature and the samples were loaded on a $5 \%$ acrylamide gel (29:1 acrylamide/bis-acrylamide; $0.5 \times$ Tris Borate EDTA).

\section{DNA transfections and CAT assays}

CEF cells were used freshly prepared according to standard methods and transfected with the $\mathrm{Ca}_{3}\left(\mathrm{PO}_{4}\right)_{2}$ precipitate technique. In addition to the expression vectors or gene reporters described in each figure, all transfections contained $100 \mathrm{ng}$ of the $\beta$-gal actosidase reporter CM V- $\beta$-gal as an internal standard and the amount of DN A was scal ed up to $10 \mu \mathrm{g}$ with pBluescript SK+DN A as carrier. For analysis of the transactivation, the cells were recovered after scraping and centrifugation in cold PBS and lysed in $100 \mu \mathrm{l}$ of $0.25 \mathrm{M}$ Tris- $\mathrm{HCl}(\mathrm{pH} \mathrm{7.5)}$ by three cycles of freeze-thawing. For each transfection, a $10 \mu \mathrm{l}$ sample was analyzed for $\beta$-galactosidase activity and a volume containing a defined amount of activity was then assayed for CAT activity. The reactions were done twice for $1 \mathrm{hr}$ (reporter $\mathrm{p}_{\alpha} \mathrm{D}_{3}$ ) and twice for $20 \mathrm{~min}$ (reporter $17 \mathrm{m5}$-TATA-CAT) at $37^{\circ} \mathrm{C}$. They were then analyzed by standard thin-layer chromatography and after autoradiography, the conversion percentage was determined by a quantitative phospholmager using a Fujix BA S 2000 apparatus. The results from three independent experiments performed with two independent DNA preparations were combined to determine the mean activities shown in the figures.

DNA transfections, immunoprecipitations, GST fusion protein selection, and Western blot analysis

Cos-7 cells, grown in 5\% calf serum-supplemented Dulbecco's medium (Sigma Chemical, St. Louis, MO), were transfected like the CEF cells with recombinant adjusted to $10 \mu \mathrm{g}$ with pBluescript SK+as carrier DN A. After $36 \mathrm{hr}$, the cells were harvested in cold PBS, pelleted, washed, and resuspended in lysis buffer [400 mM KCl, $20 \mathrm{~mm}$ Tris- $\mathrm{HCl}$ (pH 7.5), 20\% glycerol, $5 \mathrm{~mm}$ DTT, $0.4 \mathrm{~mm} \mathrm{PMSF]} \mathrm{containing} 2.5 \mathrm{mg} / \mathrm{ml}$ of leupeptin, pepstatin, aprotinin, antipain, and chymostatin. After three cycles of freeze-thaw in liquid nitrogen, the resulting cell lysate was diluted four times with the lysis buffer without $\mathrm{KCl}$ to give a final concentration of $100 \mathrm{~mm} \mathrm{KCl}$ and then cleared by centrifugation for $5 \mathrm{~min}$ at $13,000 \mathrm{rpm}$. The protein concentration was determined by the Bradford assay.

Two hundred fifty micrograms of protein extract, adjusted to $1 \mathrm{ml}$ with the $100 \mathrm{~mm} \mathrm{KCl}$ lysis buffer, was incubated for $2 \mathrm{hr}$ in a coldroom with agitation either with gl utathione-agarose or with protein G-Sepharose in the presence of the B10 monoclonal antibody produced in mouse and raised against the $B$ epitope of the estrogen receptor. The Sepharose beads were then recovered by centrifugation and washed three times with $1 \mathrm{ml}$ of RIPA buffer ( $1 \times$ PBS, $0.1 \%$ SDS, $0.5 \%$ sodium deoxycholate, $0.5 \%$ N P-40). The adsorbed proteins were dissociated by boiling for $5 \mathrm{~min}$ in $20 \mu \mathrm{l}$ of Laemmli buffer and resolved by SDSpolyacrylamide gel electrophoresis. Proteins separated by electrophoresis were electrotransferred onto a nitrocellulose filter. Blocking, washing, and incubation of the membrane with antibodies were carried out in $1 \times$ PBS containing $5 \%$ skimmed dry milk and $0.5 \%$ Tween 20 . As the proteins in our study have different molecular masses, the filter was cut and each part was probed with the appropriate antibody. We used the 2B8 monoclonal antibody (that recognizes an epitope in the carboxy-terminal part of Pnr) to detect Pnr and Pnr ${ }^{\mathrm{D}}$, the B10 monoclonal antibody for the B-tagged Ush, and the 1D10 monoclonal antibody, which recognizes the GST part of the fusion proteins. After washing ( $1 \times$ PBS, $0.5 \%$ Tween 20$)$ and blocking ( $1 \times$ PBS, $5 \%$ skimmed dry milk, $0.5 \%$ Tween 20 ), the blot was further incubated with horseradish peroxidase-linked goat anti-mouse immunoglobulins Jackson ImmunoResearch Laboratories, West Grove, PA). Specific immunocomplexes were visualized by the enhanced chemiluminescence (ECL) Western blotting detection system according to the recommendations of the manufacturer (Amersham International, Les Ulis, France).

Yeast interaction assays

Y east interaction assays were performed essentially as described previously (Finley and Brent 1994). Briefly, the yeast strain EGY48 with an integrated leu2 reporter gene and upstream LexA operators was transformed with pEG202-Pnr ${ }^{+}$, pEG202$\mathrm{Pnr}^{\mathrm{D} 1 / \mathrm{D} 4}$, or $\mathrm{pEG} 202-\mathrm{Pnr}^{\mathrm{VX1} / \mathrm{VX} 4}$, which allows expression of the full-length wild-type Pnr or one of its dominant versions, fused to the LexA-DBD. The different strains were then transformed with the pJG4-5-Ush plasmid, which allows galactosedependent expression of the fusion protein containing in its amino-terminal moiety the bacterial B42 activation domain efficient also in yeast. After selection on a medium lacking histidine and tryptophane and selecting for the presence of the pEG202 and pJG4-5 plasmids, the transformed yeasts were plated on a medium lacking histidine, trytophane, and leucine, with either glucose, where the B42Ush fusion protein is repressed, or with galactose, where its expression is induced. Levels of LexA fusion proteins in the different strains were monitored by Western blots using the Pnr-specific monoclonal antibody $2 \mathrm{~B} 8$.

Pnr and $U$ sh overexpression and staining for $\beta$-galactosidase activity

The wild-type pnr cDNA as well as the dominant forms pnr ${ }^{\mathrm{D} 4}$ 
and $\mathrm{pnr}^{\mathrm{V} \times 4}$ isolated from the pBluescript derivatives were subcloned in the appropriate restriction sites of the plasmid pUAST, which contains several binding sites for the GAL4 activator upstream to the basal hsp70 promoter sequences. The resulting pU AST-Pnr plasmids were used to transform embryos of a $w^{1118}$ stock. Transgenic strains were established and crossed with the driver pnr ${ }^{\mathrm{MD} 237}$, an enhancer trap line where a GAL4-containing transposon is inserted at the pnr locus, giving an imaginal expression indistinguishable from that of the pnr gene. They were also crossed with DC-enhancer-sc-lacZ; pnr ${ }^{\mathrm{MD} 237} / \mathrm{TM} 6 \mathrm{~B}, \mathrm{~Tb}$ flies. The transgenic lines DC-3.2 harbor the 5.7-kb EcoRI fragment that contains the dorsocentral enhancer fragment fused to $3.7 \mathrm{sc}-\mathrm{lacZ}$ (3.7 kb of the sc promotor regi on fused to the lacZ gene; for details see Gomez-Skarmeta et al. 1995). The transgenic line containing the UAS-Ush construct is described in Cubadda et al. (this issue). Larvae were dissected and discs stained for $\beta$-galactosidase activity according to standard methods.

\section{Acknowledgments}

We thank Gary Felsenfeld for the gift of the GATA expression vectors and the globin reporter, Juan Modolell for his continuous help with ac-lacZ transgenic flies, Roger Brent and Lauren $\mathrm{Ha}$ for kindly providing us with the material as well as Zeev Paroush for his help in setting up the yeast two-hybrid assay, Mariann Bienz for making the UAS-Pnr flies, Irwin Davidson for the gift of plasmids, the sequencing, oligonucleotide synthesis, and cell culture services of the IGBMC, Stuart Orkin for sharing unpublished information with us, our colleagues at the IGBM C for their help and discussions, and Irwin Davidson for comments on the manuscripts. This work was supported by the the IN SERM, the CNRS, the Centre Hospitalier Universitaire Régional, grant 92N 60/0694 from the MRE, the Association pour la Recherche Contre le Cancer, the European Community (contract ERBCHRXCT 940692), and the Action Concertées-Sciences du Vivant $\mathrm{N}^{\circ} 4$ du Ministère de l'Education $\mathrm{N}$ ationale de I'Enseignement Supérieur et de la Recherche.

The publication costs of this article were defrayed in part by payment of page charges. This article must therefore be hereby marked "advertisement" in accordance with 18 USC section 1734 solely to indicate this fact.

\section{References}

Abel, T., A.M. Michelson, and T. Maniatis. 1993. A Drosophila GATA family member that binds to Adh regulatory sequences is expressed in the developing fat body. Development 119: 623-633.

Andrews, N.C. and S.H. Orkin. 1994. Transcriptional control of erythropoiesis. Curr. O pin. Hematol. 1: 119-124.

Brand, A.H. and N . Perrimon. 1993. Targeted gene expression as a means of altering cell fates and generating dominant phe notypes. Development 118: 401-415.

Calleja, M., E. Moreno, S. Pelaz, and G. Morata. 1996. Visualization of gene expression in living adult Drosophila. Science 274: 252-255.

Campuzano, S. and J. Modolell. 1992. Patterning of the Drosophila nervous system: The achaete-scute gene complex. Trends Genet. 8: 202-208.

Chatton, B., A. Bahr, J. Acker, and C. Kedinger. 1995. Eukaryotic GST fusion vector for the study of protein-protein interactions in vivo: Application to interaction of ATFa with Jun and Fos. BioTechniques 18: 142-145.
Crossley, M. and S.H. Orkin. 1993. Regulation of the $\beta$-globin locus. Curr. Opin. Genet. Dev. 3: 232-237.

Crossley, M., M. Merika, and S.H. Orkin. 1995. Self-association of the erythroid transcription factor GATA-1 mediated by its zinc finger domains. Mol. Cell. Biol. 15: 2448-2456.

Cubadda, Y., P. Heitzler, R.P. Ray, M. Bourouis, P. Ramain, W. Gelbart, P. Simpson, and M. Haenlin. 1997. U-shaped encodes a zinc finger protein that regulates the proneural genes achaete and scute during the formation of bristles in Drosophila. Genes \& Dev. (this issue).

Cubas, P. and J. Modolell. 1992. The extramacrochaetae gene provides information for sensory organ patterning. EMBO J. 11: 3385-3393.

Cubas, P., J.F. de Celis, S. Campuzano, and J. Modolell. 1991. Proneural clusters of achaete-scute expression and the generation of sensory organs in the Drosophila imaginal disc. Genes \& Dev. 5: 996-1008.

Cunninngham, T.S. and T.G. Cooper. 1991. Expression of the DAL80 gene, whose product is homologous to the GATA factors and is a negative regulator of multiple nitrogen catabolic genes in Saccharomyces cerevisiae, is sensitive to nitrogen catabolic repression. Mol. Cell. Biol. 11: 6205-6215.

deBoer, E., M. Antoniou, V. Mignotte, L. Wall, and F. Grosveld. 1988. The human $\beta$-gl obin promoter: $\mathrm{N}$ ucl ear protein factors and erythroid specific induction of transcription. EMBO J. 7: 4203-4212.

Evans, T. and G. Felsenfeld. 1988. An erythrocyte-specific DN A-binding factor recognizes a regulatory sequence common to all chicken globin genes. Proc. Natl. Acad. Sci. 85: 5976-5980.

-_- 1989. The erythroid-specific transcription factor Eryf1: A new finger protein. Cell 58: 877-885.

- - 1 . 1991. Trans-activation of a gl obin promoter in non erythroid cells. Mol. Cell. Biol. 11: 843-853.

Finley, R.L., Jr. and R.Brent. 1994. Interaction trap cloning with yeast. In Gene probes: a pratical approach. (ed. D. Hames and D. Glover).

Ghysen, A. and C. Dambly-Chaudière. 1988. From DNA to form: The achaete-scute complex. Genes \& Dev. 2: 495-501.

Gyuris, J., E. Golemis, H. Chertkov, and R. Brent. 1993. Cdi 1, a human G1-phase and S-phase protein phosphatase that associates with cdk2. Cell 75: 791-803.

Gomez-Skarmeta, J.L., I. Rodriguez, C. Martinez, J. Culi, D. Ferres-M arco, D. Beamonte, and J. M odolell. 1995. Cis-regulation of achaete and scute: Shared enhancer-like elements drive their expression in proneural clusters of the imaginal discs. Genes \& Dev. 9: 1869-1882.

Heitzler, P. 1993. "La détermination des précurseurs nerveux chez la Drosophile: Interactions cellulaires et contrôle géné tique". Thesis. Université Louis Pasteur, Strasbourg, France.

Heitzler, P., M. Haenlin, P. Ramain, M. Calleja, and P. Simpson. 1996. A genetic analysis of pannier, a gene necessary for viability of dorsal tissues and bristle positioning in Drosophila. Nature Genet. 143: 1271-1286.

Higgs, D.R. and W.G. Wood. 1993. Understanding erythroid differentiation. Curr. Biol. 3: 548-550.

Hwang, J.J., P. Chambon, and I. Davidson. 1993. Characterization of the transcription activation domain function and the DNA binding domain of transcriptional enhancer factor-1. EMBO J. 12: 2337-2348.

Kudla, B., M.X. Caddick, T. Langdon, N .M. M artinez-Rossi, C.F. Bennett, S. Sibley, R.W. Davies, and H.N. Arst, Jr. 1990. The regulatory gene areA mediating nitrogen metabolite repression in Aspergillus nidulans. Mutations affecting specificity of gene activation alter a loop residue of a putative zinc finger. EMBO J. 9: 1355-1364. 
Laverierre, A.C., C. MacN eill, R.E. Mueller, J.B. Poelmann, E. Burch, and T. Evans. 1994. GATA 4/5/6: A subfamily of three transcription factors expressed in developing heart and gut. J. Biol. Chem. 269: 23177-23184.

Lin, W.H., L.H. Huang, J.Y. Yeh, J. Hoheisel, H. Lehrach, Y.H. Sun, and S.F. Tsai. 1995. Expression of a Drosophila GATA transcription factor in multiple tissues in developing embryos. J. Biol. Chem. 270: 25150-25158.

Merika, M. and S.H. Orkin. 1995. Functional synergy and physical interactions of the erythroid transcription factor GATA-1 with the Krüppel family proteins Spl and EKLF. Mol. Cell. Biol. 15: 2437-2447.

Mignotte, V., J.F. Eleouet, N. Raich, and P.H. Roméo. 1989a. Cis- and trans-acting elements involved in the regulation of the erythroid promoter of the human porphobilinogen deaminase gene. Proc. Natl. Acad. Sci. 86: 6548-6552.

Mignotte, V., L. Wall, E. deBoer, F. Grosveld, and P.H. Roméo. 1989b. Two tissue-specific factors bind the erythroid promoter of the human porphobilinogen deaminase. Nucleic Acids Res. 17: 37-54.

Miller, I.J. and J.J. Bieker. 1993. A novel erythroid cell-specific murine transcription factor that binds to the CACCC element and is rel ated to the Krüppel family of nuclear proteins. Mol. Cell. Biol. 13: 2776-2786.

Orkin, S.H. 1992. GATA-binding transcription factors in hematopoietic cells. Blood 80: 575-581.

Nicolis, S., C. Bertini, A. Ronchi, S. Crotta, L. Lanfranco, E. Moroni, B. Giglioni, and S. Ottolenghi. 1991. An erythroid specific enhancer upstream to the gene encoding the cellspecific transcription factor GATA-1. Nucleic Acids Res. 19: 5285-5291.

Pedone, P.V., J.G. Omichinski, P. Nony, C. Trainor, A.M. Gronenborn, G.M. Clore, and G. Felsenfeld. 1997. The Nterminal fingers of chicken GATA-2 and GATA-3 are independent sequence-specific DNA binding domains. EMBO J. 16: 2874-2882.

Pevny, L., M.C. Simon, E. Robertson, W.H. Klein, S.F. Tsai, Y. D'A gati, S.H. Orkin, and F. Constantini. 1991. Erythroid differentiation in chimeric mice blocked by a targeted mutation in the gene for transcription factor GATA-1. Nature 349: 257-260.

Philipsen, S., S. Pruzina, and F. Grosveld. 1993. The minimal requirement for activity in transgenic mice of hypersensitive site $3^{\prime}$ of the $\beta$-globin locus control region. EMBO J. 12: 1077-1085.

Plumb, M.A., J. Frampton, H. Wainwright, M. Walker, K. Macleod, G. Goodwin, and P. Harrison. 1989. GATAAG: A cis control region binding an erythroid-specific nuclear factor with a role in globin and non globin gene expression. Nucleic Acids Res. 17: 73-91.

Rahuel, C., M.A. Vinit, V. Lemarchandel, J.P. Cartron, and P.H. Roméo. 1992. Erythroid specific activity of the glycophorin promoter requires GATA-1 mediated displacement of a repressor. EMBO J. 11: 4095-4102.

Ramain, P., P. Heitzler, M. Haenlin, and P. Simpson. 1993. Pannier, a negative regulator of achaete and scute in Drosophila, encodes a zinc finger protein with homology to the vertebrate transcription factor GATA-1. Development 119: 12771291.

Romani, S., S. Campuzano, E.R. Macagno, and J. Modolell. 1989. Expression of achaete and scute genes in Drosophila imaginal discs and their function in sensory organ development. Genes \& Dev. 3: 997-1007.

Sambrook, J., E.F. Fritsch, and T. Maniatis. 1989. Molecular cloning: A laboratory manual, 2nd ed. Cold Spring Harbor Laboratory Press, Cold Spring Harbor, NY.
Simon, M.C., L. Pevny, M.V. Wiles, G. Keller, F. Constantini, and S.H. Orkin. 1992. Rescue of erythroid development in gene targeted GATA-1 mouse embryonic stem cells. Nature Genet. 1: 92-98.

Skeath, J.B. and S. Carroll. 1991. Regulation of achaete-scute gene expression and sensory organ pattern formation in the Drosophila wing. Genes \& Dev. 5: 984-995.

Spieth, J.Y., H. Shim, K. Lea, R. Conrad, and T. Blumenthal. 1991. Elt-1, an embryonically expressed Caenorhabditis elegans gene homologous to the GATA transcription factor family. Mol. Cell. Biol. 11: 4651-4659.

Tsai, S.F., D.I.K. Martin, L.I. Zon, A.D. D'Andrea, G.G. Wong, and S.H. Orkin. 1989. Cloning of CDNA for the major DNAbinding protein of the erythroid lineage through expression cloning in mammalian cells. Nature 339: 446-451.

Tsai, S.F., E. Strauss, and S.H. Orkin. 1991. Functional analysis and in vivo footprinting implicate the erythroid transcription factor GATA-1 as a positive regulator of its own promoter. Genes \& Dev. 5: 919-931.

Tsang, A.P., J.E. Visvader, C.A. Turner, Y. Fujiwara, C. Yu, M.J. Weiss, M. Crossley, and S.H. Orkin. 1997. FOG, a novel multitype zinc finger protein, acts as a co-factor for transcription factor GATA-1 in erythroid and megakaryotic differentiation. Cell (in press).

Villares, R. and C. Cabrera. 1987. The achaete-scute gene complex of D. melanogaster: Conserved domains in a subset of genes required for neurogenesis and their homology to myc. Cell 50: 415-424.

Wall, L., E. deBoer, and F. Grosveld. 1988. The human $\beta$-globin gene $3^{\prime}$ enhancer contains multiple binding sites for an erythroid-specific protein. Genes \& Dev. 2: 1089-1100.

Whyatt, D.J., E. deBoer, and F. Grosveld. 1993. The two zinc finger-like domains of GATA-1 have different DNA binding specificities. EMBO J. 12: 4993-5005.

Winick, J., T. A bel, M.W. Leonard, A.M. Michelson, I. ChardonLoriaux, R.A. Holmgren, T. M aniatis, and J.D. Engel. 1993. A GATA family transcription factor is expressed al ong the embryonic dorso-ventral axis in Drosophila melanogaster. Development 119: 1055-1065.

Xiao, J.H., I. Davidson, J.M. Garnier, and P. Chambon. 1991. Cloning, expression and transcriptional properties of the human enhancer factor TEF-1. Cell 65: 551-568.

Yamamoto, M., L.J. Ko, M.W. Leonard, H. Beug, S.H. Orkin, and J.D. Engel. 1990. Activity and tissue-specific expression of the transcription factor $\mathrm{N}$ f-E1 multigene family. Genes \& Dev. 4: 1650-1662.

Yang, H.Y. and T. Evans. 1992. Distinct roles for the two cGATA-1 finger domains.Mol. Cell. Biol. 12:4562-4570. 


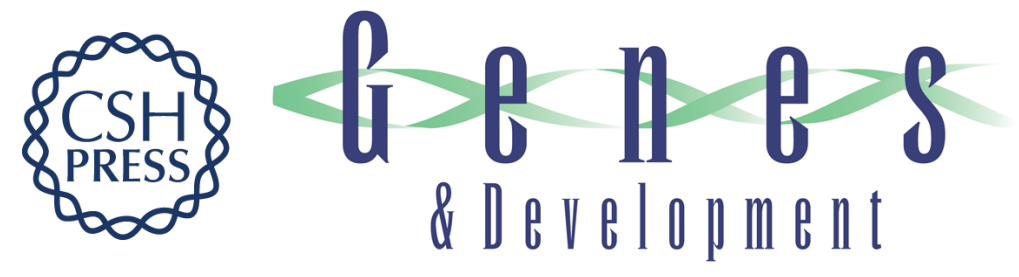

\section{Transcriptional activity of Pannier is regulated negatively by heterodimerization of the GATA DNA-binding domain with a cofactor encoded by the u-shaped gene of Drosophila}

Marc Haenlin, Yolande Cubadda, Francois Blondeau, et al.

Genes Dev. 1997, 11:

Access the most recent version at doi:10.1101/gad.11.22.3096

$\begin{array}{ll}\text { References } & \begin{array}{l}\text { This article cites } 48 \text { articles, } 25 \text { of which can be accessed free at: } \\ \text { http://genesdev.cshlp.org/content/11/22/3096.full.html\#ref-list-1 }\end{array}\end{array}$

License

Email Alerting Receive free email alerts when new articles cite this article - sign up in the box at the top Service right corner of the article or click here.

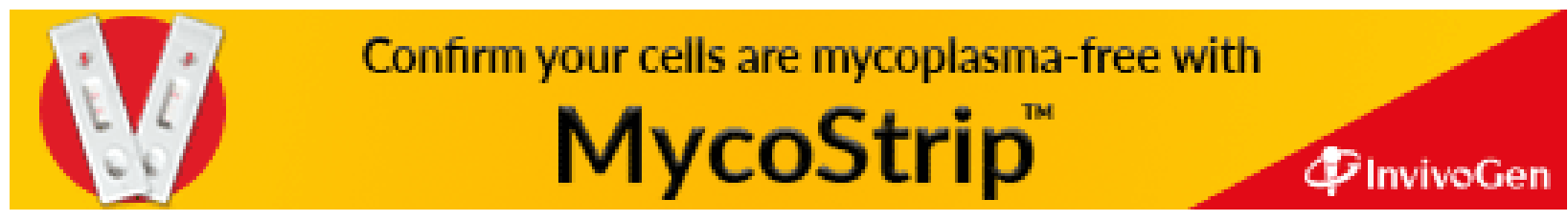

